\title{
The future of human behaviour research
}

Human behaviour is complex and multifaceted, and is studied by a broad range of disciplines across the social and natural sciences. To mark our 5th anniversary, we asked leading scientists in some of the key disciplines that we cover to share their vision of the future of research in their disciplines. Our contributors underscore how important it is to broaden the scope of their disciplines to increase ecological validity and diversity of representation, in order to address pressing societal challenges that range from new technologies, modes of interaction and sociopolitical upheaval to disease, poverty, hunger, inequality and climate change. Taken together, these contributions highlight how achieving progress in each discipline will require incorporating insights and methods from others, breaking down disciplinary silos.

\begin{abstract}
$\square$ enuine progress in understanding human behaviour can only be achieved through a multidisciplinary community effort. Five years after the launch of Nature Human Behaviour, twenty-two leading experts in some of the core disciplines within the journal's scope share their views on pressing open questions and new directions in their disciplines. Their visions provide rich insight into the future of research on human behaviour.
\end{abstract}

\section{Artificial intelligence}

\section{Kate Crawford}

Much has changed in artificial intelligence since a small group of mathematicians and scientists gathered at Dartmouth in 1956 to brainstorm how machines could simulate cognition. Many of the domains that those men discussed - such as neural networks and natural language processing - remain core elements of the field today. But what they did not address was the far-reaching social, political, legal and ecological effects of building these systems into everyday life: it was outside their disciplinary view.

Since the mid-2000s, artificial intelligence (AI) has rapidly expanded as a field in academia and as an industry, and now a handful of powerful technology corporations deploy these systems at a planetary scale. There have been extraordinary technical innovations, from real-time language translation to predicting the $3 \mathrm{D}$ structures of proteins ${ }^{1,2}$. But the biggest challenges remain fundamentally social and political: how $\mathrm{AI}$ is widening power asymmetries and wealth inequality, and creating forms of harm that need to be prioritized, remedied and regulated.

The most urgent work facing the field today is to research and remediate the costs and consequences of AI. This requires a deeper sociotechnical approach that can contend with the complex effect of AI on societies and ecologies. Although there has been important work done on algorithmic fairness in recent years ${ }^{3,4}$, not

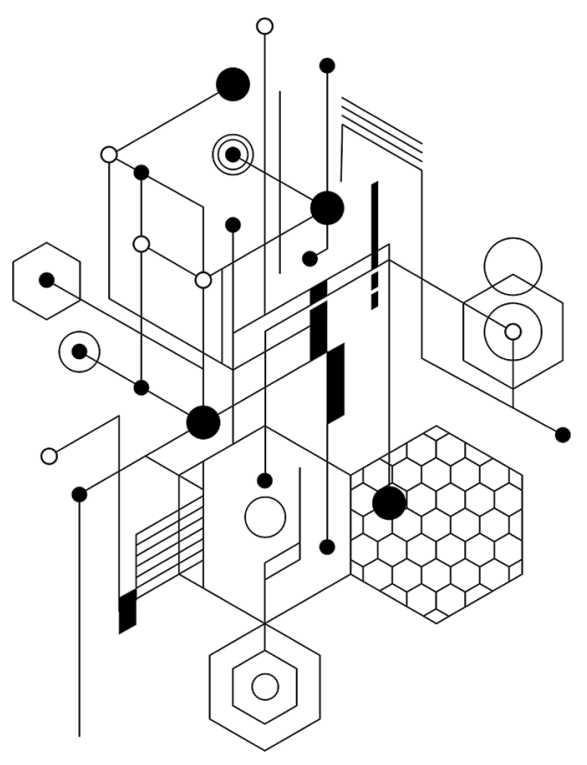

It's all connected. Credit: amtitus / DigitalVision Vectors / Getty

enough has been done to address how training data fundamentally skew how AI models interpret the world from the outset. Second, we need to address the human costs of $\mathrm{AI}$, which range from discrimination and misinformation to the widespread reliance on underpaid labourers (such as the crowd-workers who train AI systems for as little as US \$2 per hour $)^{5}$. Third, there must be a commitment to reversing the environmental costs of AI, including the exceptionally high energy consumption of the current large computational models, and the carbon footprint of building and operating modern tensor processing hardware ${ }^{6}$. Finally, we need strong regulatory and policy frameworks, expanding on the EU's draft AI Act of 2021.

By building a more interdisciplinary and inclusive AI field, and developing a more rigorous account of the full impacts of AI, we give engineers and regulators alike the tools that they need to make these systems more sustainable, equitable and just.

Kate Crawford is Research Professor at the Annenberg School, University of Southern California, Los Angeles, CA, USA; Senior Principal Researcher at Microsoft Research New York, New York, NY, USA; and the Inaugural Visiting Chair of AI and Justice at the École Normale Supérieure, Paris, France.

Anthropology

Laura M. Rival

The field of anthropology faces fundamental questions about its capacity to intervene more effectively in political debates. How can we use the knowledge that we already have to heal the imagined whole while keeping people in synchrony with each other and with the world they aspire to create for themselves and others?

The economic systems that sustain modern life have produced pernicious waste cultures. Globalization has accelerated planetary degradation and global warming through the continuous release of toxic waste. Every day, like millions of others, I dutifully clean and prepare my waste for recycling. I know it is no more than a transitory measure geared to grant manufacturers time to adjust and adapt. Reports that most waste will not be recycled, but dumped or burned, upset me deeply. How can anthropology remain a critical project in the face of such orchestrated cynicism, bad faith and indifference? How should anthropologists deploy their skills and bring a sense of shared responsibility to the task of replenishing the collective will?

To help to find answers to these questions, anthropologists need to radically rethink the ways in which we describe the processes and relations that tie communities to their environments. The extinction of experience (loss of direct contact with nature) that humankind currently suffers is massive, but not irreversible. New forms of storytelling have successfully challenged 
modernist myths, particularly their homophonic promises ${ }^{7}$. But there remain persistent challenges, such as the seductive and rampant power of one-size-fits-all progress, and the actions of elites, who thrive on emulation, and in doing so fuel run-away consumerism.

To combat these challenges, I simply reassert that 'nature' is far from having outlasted its historical utility. Anthropologists must join forces and reanimate their common exploration of the immense possibilities contained in human bodies and minds. No matter how overlooked or marginalized, these natural potentials hold the key to what keeps life going.

Laura M. Rival is Professor of Anthropology of Nature, Society and Development, ODID and SAME, University of Oxford, Oxford, UK.

\section{Communication and media studies \\ Jean Burgess}

The communication and media studies field has historically been animated by technological change. In the process, it has needed to navigate fundamental tensions: communication can be understood as both transmission (of information), and as (social) ritual ${ }^{8}$; relatedly, media can be understood as both technology and as culture? .

The most important technological change over the past decade has been the 'platformization' ${ }^{10}$ of the media environment. Large digital platforms owned by the world's most powerful technology companies have come to have an outsized and transformative role in the transmission (distribution) of information, and in mediating social practices (whether major events or intimate daily routines). In response, digital methods have transformed the field. For example, advances in computational techniques enabled researchers to study patterns of communication on social media, leading to disciplinary trends such as the quantitative description of 'hashtag publics' in the mid-2010s ${ }^{11}$.

Platforms' uses of data, algorithms and automation for personalization, content moderation and governance constitute a further major shift, giving rise to new methods (such as algorithmic audits) that go well beyond quantitative description ${ }^{12}$. But platform companies have had a patchy - at times hostile - relationship to independent research into their societal role, leading to data lockouts and even public attacks on researchers. It is important in the interests of public oversight and open science that we coordinate responses to such attempts to suppress research ${ }^{13,14}$.
As these processes of digital transformation continue, new connections between the humanities and technical disciplines will be necessary, giving rise to a new wave of methodological innovation. This next phase will also require more hybrid (qualitative and quantitative; computational and critical) methods ${ }^{15}$, not only to get around platform lockouts but also to ensure more careful attention is paid to how the new media technologies are used and experienced in everyday life. Here, innovative approaches such as the use of data donations can both aid the 'platform observability' ${ }^{16}$ that is essential to accountability, and ensure that our research involves the perspectives of diverse audiences.

Jean Burgess is Professor of Digital Media at the School of Communication and Digital Media Research Centre (DMRC), Queensland University of Technology, Brisbane, Queensland Australia; and Associate Director at the Australian Research Council Centre of Excellence for Automated Decision-Making and Society $(A D M+S)$, Melbourne, Victoria, Australia.

\section{Computational social science Claudia Wagner}

Computational social science has emerged as a discipline that leverages computational methods and new technologies to collect, model and analyse digital behavioural data in natural environments or in large-scale designed experiments, and combine them with other data sources (such as survey data).

While the community made critical progress in enhancing our understanding about empirical phenomena such as the spread of misinformation ${ }^{17}$ and the role of algorithms in curating misinformation ${ }^{18}$, it has focused less on questions about the quality and accessibility of data, the validity, reliability and reusability of measurements, the potential consequences of measurements and the connection between data, measurement and theory.

I see the following opportunities to address these issues.

First, we need to establish privacy-preserving, shared data infrastructures that collect and triangulate survey data with scientifically motivated organic or designed observational data from diverse populations ${ }^{19}$. For example, longitudinal online panels in which participants allow researchers to track their web browsing behaviour and link these traces to their survey answers will not only facilitate substantive research on societal questions but also enable methodological research (for example, on the quality of different data sources and measurement models), and contribute to the reproducibility of computational social science research.

Second, best practices and scientific infrastructures are needed for supporting the development, evaluation and re-use of measurements and the critical reflection on potentially harmful consequences of measurements ${ }^{20}$. Social scientists have developed such best practices and infrastructural support for survey measurements to avoid using instruments for which the validity is unclear or even questionable, and to support the re-usability of survey scales. I believe that practices from survey methodology and other domains, such as the medical industry, can inform our thinking here.

Finally, the fusion of algorithmic and human behaviour invites us to rethink the various ways in which data, measurements and social theories can be connected ${ }^{20}$. For example, product recommendations that users receive are based on measurements of users' interests and needs: however, users and measurements are not only influenced by those recommendations, but also influence them in turn. As a community we need to develop research designs and environments that help us to systematically enhance our understanding of those feedback loops.

Claudia Wagner is Head of Computational Social Science Department at GESIS Leibniz Institute for the Social Sciences, Köln, Germany; Professor for Applied Computational Social Sciences at RWTH Aachen University, Aachen, Germany; and External Faculty Member of the Complexity Science Hub, Vienna, Austria.

\section{Criminology}

\section{Daniel S. Nagin}

Disciplinary silos in path-breaking science are disappearing. Criminology has had a longstanding tradition of interdisciplinarity, but mostly in the form of an uneasy truce of research from different disciplines appearing side-by-side in leading journals - a scholarly form of parallel play. In the future, this must change because the big unsolved challenges in criminology will require cooperation among all of the social and behavioural sciences.

These challenges include formally merging the macro-level themes emphasized by sociologists with the micro-, individual-level themes emphasized by psychologists and economists. Initial steps have been made by economists who apply game theory to model crime-relevant social 
interactions, but much remains to be done in building models that explain the formation and destruction of social trust, collective efficacy and norms, as they relate to legal definitions of criminal behaviour.

A second opportunity concerns the longstanding focus of criminology on crimes involving the physical taking of property and interpersonal physical violence. These crimes are still with us, but - as the daily news regularly reports the internet has opened up broad new frontiers for crime that allow for thefts of property and identities at a distance, forms of extortion and human trafficking at a massive scale (often involving untraceable transactions using financial vehicles such as bitcoin) and interpersonal violence without physical contact. This is a new and largely unexplored frontier for criminological research that criminologists should dive into in collaboration with computer scientists who already are beginning to troll these virgin scholarly waters.

The final opportunity I will note also involves drawing from computer science, the primary home of what has come to be called machine learning. It is important that new generations of criminologists become proficient with machine learning methods and also collaborate with its creators. Machine learning and related statistical methods have wide applicability in both the traditional domains of criminological research and new frontiers. These include the use of prediction tools in criminal justice decision-making, which can aid in crime detection, and the prevention and measuring of crime both online and offline, but also have important implications for equity and fairness due to their consequential nature.

Daniel S. Nagin is Teresa and H. John Heinz III University Professor of Public Policy and Statistics at the Heinz College of Information Systems and Public Policy, Carnegie Mellon University, Pittsburgh, PA, USA.

\section{Behavioural economics}

\section{Bertil Tungodden}

Behavioural and experimental economics have transformed the field of economics by integrating irrationality and nonselfish motivation in the study of human behaviour and social interaction. A richer foundation of human behaviour has opened many new exciting research avenues, and I here highlight three that I find particularly promising.

Economists have typically assumed that preferences are fixed and stable, but a growing literature, combining field and laboratory experimental approaches, has provided novel evidence on how the social environment shapes our moral and selfish preferences. It has been shown that prosocial role models make people less selfish $^{21}$, that early-childhood education affects the fairness views of children ${ }^{22}$ and that grit can be fostered in the correct classroom environment ${ }^{23}$. Such insights are important for understanding how exposure to different institutions and socialization processes influence the intergenerational transmission of preferences, but much more work is needed to gain systematic and robust evidence on the malleability of the many dimensions that shape human behaviour.

The moral mind is an important determinant of human behaviour, but our understanding of the complexity of moral motivation is still in its infancy. A growing literature, using an impartial spectator design in which study participants make consequential choices for others, has shown that people often disagree on what is morally acceptable. An important example is how people differ in their view of what is a fair inequality, ranging from the libertarian fairness view to the strict egalitarian fairness view ${ }^{24,25}$. An exciting question for future research is whether such moral differences reflect a concern for other moral values, such as freedom, or irrational considerations.

A third exciting development in behavioural and experimental economics is the growing set of global studies on the foundations of human behaviour $^{26,27}$. It speaks to the major concern in the social sciences that our evidence is unrepresentative and largely based on studies with samples from Western, educated, industrialized, rich and democratic societies ${ }^{28}$. The increased availability of infrastructure for implementing large-scale experimental data collections and methodological advances carry promise that behavioural and experimental economic research will broaden our understanding of the foundations of human behaviour in the coming years.

Bertil Tungodden is Professor and Scientific Director of the Centre of Excellence FAIR at NHH Norwegian School of Economics, Bergen, Norway.

\section{Development economics \\ Esther Duflo}

The past three decades have been a wonderful time for development economics. The number of scholars, the number of publications and the visibility of the work has dramatically increased. Development economists think about education, health, firm growth, mental health, climate, democratic rules and much more. No topic seems off limits!

This progress is intimately connected with the explosion of the use of randomized controlled trials (RCTs) and, more generally, with the embrace of careful causal identification. RCTs have markedly transformed development economics and made it the field that it is today.

The past three decades (until the COVID-19 crisis) have also been very good for improving the circumstances of low-income people around the world: poverty rates have fallen; school enrolment has increased; and maternal and infant mortality has been halved. Although I would not dare imply that the two trends are causally related, one of the reasons for these improvements in the quality of life - even in countries where economic growth has been slow - is the greater focus on pragmatic solutions to the fundamental problems faced by people with few resources. In many countries, development economics researchers (particularly those working with RCTs) have been closely involved with policy-makers, helping them to develop, implement and test these solutions. In turn, this involvement has been a fertile ground for new questions, which have enriched the field.

I imagine future change will, once again, come from an unexpected place. One possible driver of innovation will come from this meeting between the requirements of policy and the intellectual ambition of researchers. This means that the new challenges of our planet must (and will) become the new challenges of development economics. Those challenges are, I believe, quite clear: rethinking social protection to be better prepared to face risks such as the COVID-19 pandemic; mitigating, but unfortunately also adapting to, climate changes; curbing pollution; and addressing gender, racial and ethnic inequality.

To address these critical issues, I believe the field will continue to rely on RCTs, but also start using more creatively (descriptively or in combination with RCTs) the huge amount of data that is increasingly available as governments, even in poor countries, digitize their operations. I cannot wait to be surprised by what comes next.

\section{Esther Duflo is The Abdul Latif Jameel Professor of Poverty Alleviation and Development Economics at the Department of Economics, Massachusetts Institute of Technology, Cambridge MA, USA; and}


cofounder and codirector of the Abdul Latif Jameel Poverty Action Lab (J-PAL).

\section{Political science}

Janet M. Box-Steffensmeier

Political science remains one of the most pluralistic disciplines and we are on the move towards engaged pluralism. This takes us beyond mere tolerance to true, sincere engagement across methods, methodologies, theories and even disciplinary boundaries. Engaged pluralism means doing the hard work of understanding our own research from the multiple perspectives of others.

More data are being collected on human behaviour than ever before and our advances in methods better address the inherent interdependencies of the data across time, space and context. There are new ways to measure human behaviour via text, image and video. Data creation can even go back in time. All these advancements bode well for the potential to better understand and predict behaviour. This 'data century' and 'golden age of methods' also hold the promise to bridge, not divide, political science, provided that there is engaged methodological pluralism. Qualitative methods provide unique insights and perspectives when joined with quantitative methods, as does a broader conception of the methodologies underlying and launching our research.

I remain a strong proponent of leveraging dynamics and focusing on heterogeneity in our research questions to advance our disciplines. Doing so brings in an explicit perspective of comparison around similarity and difference. Our questions, hypotheses and theories are often made more compelling when considering the dynamics and heterogeneity that emerges when thinking about time and change.

Striving for a better understanding of gender, race and ethnicity is driving deeper and fuller understandings of central questions in the social sciences. The diversity of the research teams themselves across gender, sex, race, ethnicity, first-generation status, religion, ideology, partisanship and cultures also pushes advancement. One area that we need to better support is career diversity. Supporting careers in government, non-profit organizations and industry, as well as academia, for graduate students will enhance our disciplines and accelerate the production of knowledge that changes the world.

Engaged pluralism remains a foundational key to advancement in political science. Engaged pluralism supports critical diversity, equity and inclusion work, strengthens political scientists' commitment to democratic principles, and encourages civic engagement more broadly. It is an exciting time to be a social scientist.

Janet M. Box-Steffensmeier is Vernal Riffe Professor of Political Science, Professor of Sociology (courtesy) and Distinguished University Professor at the Department of Political Science, Ohio State University, Columbus OH, USA; and immediate past President of the American Political Science Association.

\section{Cognitive psychology}

\section{Andrew Perfors}

Cognitive psychology excels at understanding questions whose problem-space is well-defined, with precisely specified theories that transparently map onto thoroughly explored experimental paradigms. That means there is a vast gulf between the current state of the art and the richness and complexity of cognition in the real world. The most exciting open questions are about how to bridge that gap without sacrificing rigour and precision. This requires at least three changes.

First, we must move beyond typical experiments. Stimuli must become less artificial, with a naturalistic structure and distribution. Similarly, tasks must become more ecologically valid: less isolated, with more uncertainty, embedded in natural situations and over different time-scales.

Second, we must move beyond considering individuals in isolation. We live in a rich social world and an environment that is heavily shaped by other humans. How we think, learn and act is deeply affected by how other people think and interact with us; cognitive science needs to engage with this more.

Third, we must move beyond the metaphor of humans as computers. Our cognition is deeply intertwined with our emotions, motivations and senses. These are more than just parameters in our minds; they have a complexity and logic of their own, and interact in nontrivial ways with each other and more typical cognitive domains such as learning, reasoning and acting.

How do we make progress on these steps? We need reliable real-world data that are comparable across people and situations, reflect the cognitive processes involved and are not changed by measurement. Technology may help us with this, but challenges surrounding privacy and data quality are huge. Our models and analytic approaches must also grow in complexity commensurate with the growth in problem and data complexity - without becoming intractable or losing their explanatory power.

Success in this endeavour calls for a different kind of science that is not centred around individual laboratories or small stand-alone projects. The biggest advances will be achieved on the basis of large, rich, real-world datasets from different populations, created and analysed in collaborative teams that span multiple domains, fields and approaches. This requires incentive structures that reward team-focused, slower science and prioritize the systematic construction of reliable knowledge over splashy findings.

Andrew Perfors is Associate Professor and Deputy Director of the Complex Human Data Hub, University of Melbourne, Melbourne, Victoria, Australia.

\section{Cultural and social psychology \\ Ying-yi Hong}

I am writing this at an exceptional moment in human history. For two years, the world has faced the COVID-19 pandemic and there is no end in sight. Cultural and social psychology are uniquely equipped to understand the COVID-19 pandemic, specifically examining how people, communities and countries are dealing with this extreme global crisis - especially at a time when many parts of the world are already experiencing geopolitical upheaval.

During the pandemic, and across different nations and regions, a diverse set of strategies (and subsequent levels of effectiveness) were used to curb the spread of the disease. In the first year of the pandemic, research revealed that some cultural worldviews - such as collectivism (versus individualism) and tight (versus loose) norms - were positively associated with compliance with COVID-19 preventive measures as well as with fewer infections and deaths ${ }^{29,30}$. These worldview differences arguably stem from different perspectives on abiding to social norms and prioritizing the collective welfare over an individual's autonomy and liberty. Although in the short term it seems that a collectivist or tight worldview has been advantageous, it is unclear whether this will remain the case in the long term. Cultural worldviews are 'tools' that individuals use to decipher the meaning of their environment, and are dynamic rather than static ${ }^{31}$. Future research can examine how cultural worldviews and global threats co-evolve.

The pandemic has also amplified the demarcation of national, political and other major social categories. On the one hand, identification with some groups (for example, national identity) was found to increase in-group care and thus a greater willingness to sacrifice personal autonomy to comply with COVID-19 measures ${ }^{32}$. On the other hand, identification with other groups (for example, political parties) 
widened the ideological divide between groups and drove opposing behaviours towards COVID-19 measures and health outcomes $^{33}$. As we are facing climate change and other pressing global challenges, understanding the role of social identities and how they affect worldviews, cognition and behaviour will be vital. How can we foster more inclusive (versus exclusive) identities that can unite rather than divide people and nations?

Ying-yi Hong is Choh-Ming Li Professor of Management and Associate Dean (Research) at the Department of Management, The Chinese University of Hong Kong, Hong Kong Special Administrative Region, China.

\section{Developmental psychology \\ Alison Gopnik}

Developmental psychology is similar to the kind of book or band that, paradoxically, everyone agrees is underrated. On the one hand, children and the people who care for them are often undervalued and overlooked. On the other, since Piaget, developmental research has tackled some of the most profound philosophical questions about every kind of human behaviour. This will only continue into the future.

Psychologists increasingly recognize that the minds of children are not just a waystation or an incomplete version of adult minds. Instead, childhood is a distinct evolutionarily adaptive phase of an organism, with its own characteristic cognitions, emotions and motivations. These characteristics of childhood reflect a different agenda than those of the adult mind - a drive to explore rather than exploit. This drive comes with motivations such as curiosity, emotions such as wonder and surprise and remarkable cognitive learning capacities. A new flood of research on curiosity, for example, shows that children actively seek out the information that will help them to learn the most.

The example of curiosity also reflects the exciting prospects for interdisciplinary developmental science. Machine learning is increasingly using children's learning as a model, and developmental psychologists are developing more precise models as a result. Curiosity-based AI can illuminate both human and machine intelligence. Collaborations with biology are also exciting: for example, in work on evolutionary 'life history' explanations of the effects of adverse experiences on later life, and new research on plasticity and sensitive periods in neuroscience. Finally, children are at the cutting edge of culture, and developmental psychologists increasingly conduct a much wider range of cross-cultural studies.

But perhaps the most important development is that policy-makers are finally starting to realize just how crucial children are to important social issues. Developmental science has shown that providing children with the care that they need can decrease poverty, inequality, disease and violence. But that care has been largely invisible to policy-makers and politicians. Understanding scientifically how caregiving works and how to support it more effectively will be the most important challenge for developmental psychology in the next century.

Alison Gopnik is Professor of Psychology and Affiliate Professor of Philosophy at the Department of Psychology, University of California at Berkeley, Berkeley, CA, USA.

\section{Science of science}

\section{Cassidy R. Sugimoto}

Why study science? The goal of science is to advance knowledge to improve the human condition. It is, therefore, essential that we understand how science operates to maximize efficiency and social good. The metasciences are fields that are devoted to understanding the scientific enterprise. These fields are distinguished by differing epistemologies embedded in their names: the philosophy, history and sociology of science represent canonical metasciences that use theories and methods from their mother disciplines. The 'science of science' uses empirical approaches to understand the mechanisms of science. As mid-twentieth-century science historian Derek de Solla Price observed, science of science allows us to "turn the tools of science on science itself" 34 .

Contemporary questions in the science of science investigate, inter alia, catalysts of discovery and innovation, consequences of increased access to scientific information, role of teams in knowledge creation and the implications of social stratification on the scientific enterprise. Investigation of these issues require triangulation of data and integration across the metasciences, to generate robust theories, model on valid assumptions and interpret results appropriately. Community-owned infrastructure and collective venues for communication are essential to achieve these goals. The construction of large-scale science observatories, for example, would provide an opportunity to capture the rapidly expanding dataverse, collaborate and share data, and provide nimble translations of data into information for policy-makers and the scientific community.
The topical foci of the field are also undergoing rapid transformation. The expansion of datasets enables researchers to analyse a fuller population, rather than a narrow sample that favours particular communities. The field has moved from an elitist focus on 'success' and 'impact' to a more-inclusive and prosopographical perspective. Conversations have shifted from citations, impact factors and $h$-indices towards responsible indicators, diversity and broader impacts. Instead of asking 'how can we predict the next Nobel prize winner?', we can ask 'what are the consequences of attrition in the scientific workforce?'. The turn towards contextualized measurements that use more inclusive datasets to understand the entire system of science places the science of science in a ripe position to inform policy and propel us towards a more innovative and equitable future.

Cassidy R. Sugimoto is Professor and Tom and Marie Patton School Chair, School of Public Policy, Georgia Institute of Technology, Atlanta, GA, USA.

\section{Sociology}

Sari Hanafi

In the past few years, we have been living through times in which reasonable debate has become impossible. Demagogical times are driven by the vertiginous rise of populism and authoritarianism, which we saw in the triumph of Donald Trump in the USA and numerous other populist or authoritarian leaders in many places around the globe. There are some pressing tasks for sociology that can be, in brief, reduced to three.

First, fostering democracy and the democratization process requires disentangling the constitutive values that compose the liberal political project (personal liberty, equality, moral autonomy and multiculturalism) to address the question of social justice and to accommodate the surge in people's religiosity in many parts in the globe.

Second, the struggle for the environment is inseparable from our choice of political economy, and from the nature of our desired economic system - and these connections between human beings and nature have never been as intimate as they are now. Past decades saw rapid growth that was based on assumptions of the long-term stability of the fixed costs of raw materials and energy. But this is no longer the case. More recently, financial speculation intensified and profits shrunk, generating distributional conflicts between workers, management, owners and tax authorities. 
The nature of our economic system is now in acute crisis.

The answer lies in a consciously slow-growing new economy that incorporates the biophysical foundations of economics into its functioning mechanisms. Society and nature cannot continue to be perceived each as differentiated into separate compartments. The spheres of nature, culture, politics, social, economy and religion are indeed traversed by common logics that allow a given society to be encompassed in its totality, exactly as Marcel Mauss ${ }^{35}$ did. The logic of power and interests embodied in 'Homo economicus' prevents us from being able to see the potentiality of human beings to cultivate gift-giving practices as an anthropological foundation innate within social relationships.

Third, there are serious social effects of digitalized forms of labour and the trend of replacing labour with an automaton. Even if digital labour partially reduces the unemployment rate, the lack of social protection for digital labourers would have tremendous effects on future generations.

In brief, it is time to connect sociology to moral and political philosophy to address fundamentally post-COVID-19 challenges.

\section{Sari Hanafi is Professor of Sociology at the American University of Beirut, Beirut, Lebanon; and President of the International Sociological Association.}

\section{Environmental studies (climate change)} Yasuko Kameyama

Climate change has been discussed for more than 40 years as a multilateral issue that poses a great threat to humankind and ecosystems. Unfortunately, we are still talking about the same issue today. Why can't we solve this problem, even though scientists pointed out its importance and urgency so many years ago?

These past years have been spent trying to prove the causal relationship between an increase in greenhouse gas concentrations, global temperature rise and various extreme weather events, as well as developing and disseminating technologies needed to reduce emissions. All of these tasks have been handled by experts in the field. At the same time, the general public invested little time in this movement, probably expecting that the problem would be solved by experts and policy-makers. But that has not been the case. No matter how much scientists have emphasized the crisis of climate change or how many clean energy technologies engineers have developed, society has resisted making the necessary changes. Now, the chances of keeping the temperature rise within $1.5^{\circ} \mathrm{C}$ of pre-industrial levels - the goal necessary to minimize the effects of climate change are diminishing.

We seem to finally be realizing the importance of social scientific knowledge. People need to take scientific information seriously for clean technology to be quickly diffused. Companies are more interested in investing in newer technology and product development when they know that their products will sell. Because environmental problems are caused by human activity, research on human behaviour is indispensable in solving these problems.

Reports by the Intergovernmental Panel on Climate Change (IPCC) have not devoted many pages to the areas of human awareness and behaviour (https://www.ipcc. $\mathrm{ch} /$ ). The IPCC's Third Working Group, which deals with mitigation measures, has partially spotlighted research on institutions, as well as on concepts such as fairness. People's perception of climate change and the relationship between perception and behavioural change differ depending on the country, societal structure and culture. Additional studies in these areas are required and, for that purpose, more studies from regions such as Asia, Africa and South America, which are underrepresented in terms of the number of academic publications, are particularly needed.

\section{Yasuko Kameyama is Director, Social Systems Division, National Institute for Environmental Studies, Tsukuba, Japan.}

\section{Sustainability (food systems) \\ Mario Herrero}

The food system is in dire straits. Food demand is unprecedented, while malnutrition in all its forms (obesity, undernutrition and micronutrient deficiencies) is rampant. Environmental degradation is pervasive and increasing, and if it continues, the comfort zone for humanity and ecosystems to thrive will be seriously compromised. From bruises and shapes to sell-by dates, we tend to find many reasons to exclude perfectly edible food from our plates, whereas in other cases not enough food reaches hungry mouths owing to farming methods, pests and lack of adequate storage. These types of inequalities are common and - together with inherent perverse incentives that maintain the status quo of how we produce, consume and waste increasingly cheap and processed food they are launching us towards a disaster.

We are banking on a substantial transformation of the food system to solve this conundrum. Modifying food consumption and waste patterns are central to the plan for achieving healthier diets, while increasing the sustainability of our food system. This is also an attractive policy proposition, as it could lead to gains in several sectors. Noncommunicable diseases such as obesity, diabetes and heart disease could decline, while reducing the effects of climate change, deforestation, excessive water withdrawals and biodiversity loss, and their enormous associated - and largely unaccounted - costs.

Modifying our food consumption and waste patterns is very hard, and unfortunately we know very little about how to change them at scale. Yes, many pilots and small examples exist on pricing, procurement, food environments and others, but the evidence is scarce, and the magnitude of the change required demands an unprecedented transdisciplinary research agenda. The problem is at the centre of human agency and behaviour, embodying culture, habits, values, social status, economics and all aspects of agri-food systems. Certainly, one of the big research areas for the next decade if we are to reach the Sustainable Development Goals leaving no one behind.

Mario Herrero is Professor, Cornell Atkinson scholar and Nancy and Peter Meinig Family Investigator in the Life Sciences at the Department of Global Development, College of Agriculture and Life Sciences and Cornell Atkinson Center for Sustainability, Cornell University, Ithaca, NY, USA.

\section{Cultural evolution \\ Laurel Fogarty}

Humans are the ultimate 'cultural animals' We are innovative, pass our cultures to one another across generations and build vast self-constructed environments that reflect our cultural biases. We achieve things using our cultural capacities that are unimaginable for any other species on earth. And yet we have only begun to understand the dynamics of cultural change, the drivers of cultural complexity or the ways that we adapt culturally to changing environments. Scholars - anthropologists, archaeologists and sociologists - have long studied culture, aiming to describe and understand its staggering diversity. The relatively new field of cultural evolution has different aims, one of the most important of which is to understand the mechanics in the background - what general principles, if any, govern human cultural change?

Although the analogy of culture as an evolutionary process has been made since at least the time of Darwin ${ }^{36,37}$, cultural evolution as a robust field of study is much younger. From its beginnings with 
the pioneering work of Cavalli-Sforza \& Feldman $^{38-40}$ and Boyd \& Richerson ${ }^{41,42}$, the field of cultural evolution has been heavily theoretical. It has drawn on models from genetic evolution ${ }^{40,43-45}$, ecology ${ }^{46,47}$ and epidemiology $\mathrm{y}^{40,48}$, extending and adapting them to account for unique and important aspects of cultural transmission. Indeed, in its short life, the field of cultural evolution has largely been dominated by a growing body of theory that ensured that the fledgling field started out on solid foundations. Because it underpins and makes possible novel applications of cultural evolutionary ideas, theoretical cultural evolution's continued development is not only crucial to the field's growth but also represents some of its most exciting future work.

One of the most urgent tasks for cultural evolution researchers in the next five years is to develop, alongside its theoretical foundations, robust principles of application ${ }^{49-51}$. In other words, it is vital to develop our understanding of what we can - and, crucially, cannot - infer from different types of cultural data. Where do we draw those boundaries and how can we apply cultural evolutionary theory to cultural datasets in a principled way? The tandem development of robust theory and principled application has the potential to strengthen cultural evolution as a robust, useful and ground-breaking inferential science of human behaviour.

Laurel Fogarty is Senior Scientist at the Department of Human Behaviour, Ecology, and Culture, Max Planck Institute for Evolutionary Anthropology, Leipzig, Germany.

\section{Genetics}

\section{Aysu Okbay}

Over the past decade, research using molecular genetic data has confirmed one of the main conclusions of twin studies: all human behaviour is partly heritable ${ }^{52,53}$. Attempts at examining the link between genetics and behaviour have been met with concerns that the findings can be abused to justify discrimination - and there are good historical grounds for these concerns. However, these findings also show that ignoring the contribution of genes to variation in human behaviour could be detrimental to a complete understanding of social phenomena, given the complex ways that genes and environment interact.

Uncovering these complex pathways has become feasible only recently thanks to rapid technological progress reducing the costs of genotyping. Sample sizes in genome-wide association studies (GWAS) have risen from tens of thousands to millions in the past decade, reporting thousands of genetic variants associated with different behaviours ${ }^{54-57}$. New ways to use GWAS results have emerged, the most important one arguably being a method to aggregate the additive effects of many genetic variants into a 'polygenic index' (PGI) (also known as a 'polygenic score') that summarizes an individual's genetic propensity towards a trait or behaviour ${ }^{58,59}$. Being aggregate measures, PGIs capture a much larger share of the variance in the trait of interest compared to individual genetic variants ${ }^{60}$. Thus, they have paved the way for follow-up studies with smaller sample sizes but deeper phenotyping compared to the original GWAS, allowing researchers to, for example, analyse the channels through which genes operate ${ }^{61,62}$, how they interact with the environment ${ }^{63,64}$, and account for confounding bias and boost statistical power by controlling for genetic effects ${ }^{65,66}$.

Useful as they are, PGIs and the GWAS that they are based on can suffer from confounding due to environmental factors that correlate with genotypes, such as population stratification, indirect effect from relatives or assortative mating ${ }^{67}$. Now that the availability of genetic data enables large-scale within-family GWAS, the next big thing in behaviour genetic research will be disentangling these sources ${ }^{68}$. While carrying the progress further, it is important that the field prioritizes moving away from its currently predominant Eurocentric bias by extending data collection and analyses to individuals of non-European ancestries, as the exclusion of non-European ancestries from genetic research has the potential to exacerbate health disparities ${ }^{69}$. Researchers should also be careful to communicate their findings clearly and responsibly to the public and guard against their misappropriation by attempts to fuel discriminatory action and discourse ${ }^{70}$.

Aysu Okbay is Assistant Professor at the Department of Economics, School of Business and Economics, Vrije Universiteit Amsterdam, Amsterdam, The Netherlands.

\section{Cognitive neuroscience}

\section{Anna C. Nobre}

Since the 'decade of the brain' in the 1990s, ingenuity in cognitive neuroscience has focused on measuring and analysing brain signals. Adapting tools from statistics, engineering, computer science, physics and other disciplines, we studied activity, states, connectivity, interactions, time courses and dynamics in brain regions and networks.
Unexpected findings about the brain yielded important insights about the mind.

Now is a propitious time to upgrade the brain-mind duumvirate to a brain-mind-behaviour triumvirate. Brain and mind are embodied, and their workings are expressed through various effectors. Yet, experimental tasks typically use simple responses to capture complex psychological functions. Often, a button press - with its limited dimensions of latency and accuracy measures anticipating, focusing, evaluating, choosing, reflecting or remembering. Researchers venturing beyond such simple responses are uncovering how the contents of mind can be studied using various continuous measures, such as pupil diameter, gaze shifts and movement trajectories.

Most tasks also restrict participants' movements to ensure experimental control. However, we are learning that principles of cognition derived in artificial laboratory contexts can fail to generalize to natural behaviour. Virtual reality should prove a powerful methodology. Participants can behave naturally, and experimenters can control stimulation and obtain quality measures of gaze, hand and body movements. Noninvasive neurophysiology methods are becoming increasingly portable. Exciting immersive brain-mind-behaviour studies are just ahead.

The next necessary step is out of the academic bubble. Today the richest data on human behaviour belong to the information and technology industries. In our routines, we contribute data streams through telephones, keyboards, watches, vehicles and countless smart devices in the internet of things. These expose properties such as processing speed, fluency, attention, dexterity, navigation and social context. We supplement these by broadcasting feelings, attitudes and opinions through social media and other forums. The richness and scale of the resulting big data offer unprecedented opportunities for deriving predictive patterns that are relevant to understanding human cognition (and its disorders). The outcomes can then guide further hypothesis-driven experimentation. Cognitive neuroscience is intrinsically collaborative, combining a broad spectrum of disciplines to study the mind. Its challenge now is to move from a multidisciplinary to a multi-enterprise science.

Anna C. Nobre is Chair in Translational Cognitive Neuroscience at the Department of Experimental Psychology, University of Oxford, UK; and Director of Oxford Centre for Human Brain Activity, Wellcome Centre 
for Integrative Neuroimaging, Department of Psychiatry, University of Oxford, UK.

Social and affective neuroscience

\section{Tatia M. C. Lee}

Social and affective neuroscience is a relatively new, but rapidly developing, field of neuroscience. Social and affective neuroscience research takes a multilevel approach to make sense of socioaffective processes, focusing on macro- (for example, social environments and structures), meso- (for example, social interactions) and micro (for example, socio-affective neural processes and perceptions)-level interactions. Because the products of these interactions are person-specific, the conventional application of group-averaged mechanisms to understand the brain in a socioemotional context has been reconsidered. Researchers turn to ecologically valid stimuli (for example, dynamic and virtual reality instead of static stimuli) and experimental settings (for example, real-time social interaction $)^{71}$ to address interindividual differences in social and affective responses. At the neural level, there has been a shift of research focus from local neural activations to large-scale synchronized interactions across neural networks. Network science contributes to the understanding of dynamic changes of neural processes that reflect the interactions and interconnection of neural structures that underpin social and affective processes.

We are living in an ever-changing socioaffective world, full of unexpected challenges. The ageing population and an increasing prevalence of depression are social phenomena on a global scale. Social isolation and loneliness caused by measures to tackle the current pandemic affect physical and psychological well-being of people from all walks of life. These global issues require timely research efforts to generate potential solutions. In this regard, social and affective neuroscience research using computational modelling, longitudinal research designs and multimodal data integration will create knowledge about the basis of adaptive and maladaptive social and affective neurobehavioural processes and responses ${ }^{72-74}$. Such knowledge offers important insights into the precise delineation of brain-symptom relationships, and hence the development of prediction models of cognitive and socioaffective functioning (for example, refs. ${ }^{75,76}$ ). Therefore, screening tools for identifying potential vulnerabilities can be developed, and timely and precise interventions can be tailored to meet individual situations and needs. The translational application of social and affective neuroscience research to precision medicine (and policy) is experiencing unprecedented demand, and such demand is met with unprecedented clinical and research capabilities.

Tatia M. C. Lee is Chair Professor of Psychology at the State Key Laboratory of Brain and Cognitive Sciences and Laboratory of Neuropsychology and Human Neuroscience, The University of Hong Kong, Hong Kong Special Administrative Region, China.

\section{Neurology}

\section{Maurizio Corbetta}

Focal brain disorders, including stroke, trauma and epilepsy, are the main causes of disability and loss of productivity in the world, and carry a cumulative cost in Europe of about $€ 500$ billion per year ${ }^{77}$. The disease process affects a specific circuit in the brain by turning it off (as in stroke) or pathologically turning it on (as in epilepsy). The cause of the disabling symptoms is typically local circuit damage. However, there is now overwhelming evidence that symptoms reflect not only local pathology but also widespread (network) functional abnormalities. For instance, in stroke, an average lesion the size of a golf ball - typically alters the activity of on average $25 \%$ of all brain connections. Furthermore, normalization of these abnormalities correlates with optimal recovery of function ${ }^{78,79}$.

One exciting treatment opportunity is 'circuit-based' stimulation: an ensemble of methods (optogenetic, photoacoustic, electrochemical, magnetic and electrical) that have the potential to normalize activity. Presently, this type of therapy is limited by numerous factors, including a lack of knowledge about the circuits, the difficulty of mapping these circuits in single patients and, most importantly, a principled understanding of where and how to stimulate to produce functional recovery.

A possible solution lies in a strategy (developed with G. Deco, M. Massimini and M. Sanchez-Vivez) that starts with an in-depth assessment of behaviour and physiological studies of brain activity to characterize the affected circuits and associated patterns of functional abnormalities. Such a multi-dimensional physiological map of a lesioned brain can be then fed to biologically realistic in silico models ${ }^{80}$. A model of a lesioned brain affords the opportunity to explore, in an exhaustive way, different kinds of stimulation to normalize faulty activity. Once a suitable protocol is found it can be exported first to animal models, and then to humans. Stimulation alone will not be enough. Pairing with behavioural training (rehabilitation) will stabilize learning and normalize connections.

The ability to interface therapy (stimulation, rehabilitation and drugs) with brain signals or other kinds of behavioural sensor offers another exciting opportunity, to open the 'brain's black box'. Most current treatments in neuroscience are given with no regard to their effect on the underlying brain signals or behaviour. Giving patients conscious access to their own brain signals may substantially enhance recovery, as the brain is now in the position to use its own powerful connections and learning mechanisms to cure itself.

Maurizio Corbetta is Professor and Chair of Neurology at the Department of Neuroscience and Director of the Padova Neuroscience Center (PNC), University of Padova, Italy; and Principal Investigator at the Venetian Institute of Molecular Medicine (VIMM), Padova, Italy.

\section{Psychiatry}

\section{Merete Nordentoft}

Schizophrenia and related psychotic disorders are among the costliest and most debilitating disorders in terms of personal sufferings for those affected, for relatives and for society ${ }^{81}$. These disorders often require long-term treatment and, for a substantial proportion of the patients, the outcomes are poor. This has motivated efforts to prevent long-lasting illness by early intervention. The time around the onset of psychotic disorders is associated with an increased risk of suicide, of loss of affiliation with the labour market, and social isolation and exclusion. Therefore, prevention and treatment of first-episode psychosis will be a key challenge for the future.

There is now solid evidence proving that early intervention services can improve clinical outcomes ${ }^{82}$. This was first demonstrated in the large Danish OPUS trial, in which OPUS treatment - consisting of assertive outreach, case management and family involvement, provided by multidisciplinary teams over a two-year period - was shown to improve clinical outcomes ${ }^{83}$. Moreover, it was also cost-effective ${ }^{84}$. Although the positive effects on clinical outcomes were not sustainable after five and ten years, there was a long-lasting effect on use of supported housing facilities (indicating improved ability to live independently $)^{85}$. Later trials proved that it is possible to maintain the positive clinical outcomes by extending the services to five years or by offering a stepped care model with continued intensive care for the patients who are most impaired ${ }^{86}$. However, even though both clinical and 
functional outcomes (such as labour market affiliation) can be improved by evidence-based treatments ${ }^{82}$, a large group of patients with first-episode psychosis still have psychotic symptoms after ten years. Thus, there is still an urgent need for identification of new and better options for treatment.

Most probably, some of the disease processes start long before first onset of a psychotic disorder. Thus, identifying disease mechanisms and possibilities for intervention before onset of psychosis will be extremely valuable. Evidence for effective preventive interventions is very limited, and the most burning question - of how to prevent psychosis - is still open.

The early intervention approach is also promising also for other disorders, including bipolar affective disorder, depression, anxiety, eating disorders, personality disorders, autism and attention-deficient hyperactivity disorder.

Merete Nordentoft is Clinical Professor at the Department of Clinical Medicine, University of Copenhagen, Copenhagen, Denmark; and Principal Investigator, CORE - Copenhagen Research Centre for Mental Health, Mental Health Centre Copenhagen, Copenhagen University Hospital, Copenhagen, Denmark.

\section{Epidemiology}

\section{Gabriel M. Leung}

In a widely anthologized article from the business field of marketing, Levitt ${ }^{87}$ pointed out that often industries failed to grow because they suffered from a limited market view. For example, Kodak went bust because it narrowly defined itself as a film camera company for still photography rather than one that should have been about imaging writ large. If it had had that strategic insight, it would have exploited and invested in digital technologies aggressively and perhaps gone down the rather more successful path of Fujifilm - or even developed into territory now cornered by Netflix.

The raison d'être of epidemiology has been to provide a set of robust scientific methods that underpin public health practice. In turn, the field of public health has expanded to fulfil the much-wider and more-intensive demands of protecting, maintaining and promoting the health of local and global populations, intergenerationally. At its broadest, the mission of public health should be to advance social justice towards a complete state of health.

Therefore, epidemiologists should continue to recruit and embrace relevant methodology sets that could answer public health questions, better and more efficiently. For instance, Davey Smith and Ebrahim $^{88}$ described how epidemiology adapted instrumental variable analysis that had been widely deployed in econometrics to fundamentally improve causal inference in observational epidemiology. Conversely, economists have not been shy in adopting the randomized controlled trial design to answer questions of development, and have recognized it with a Nobel prize ${ }^{89}$. COVID-19 has brought mathematical epidemiology or modelling to the fore. The foundations of the field borrowed heavily from population dynamics and ecological theory.

In future, classical epidemiology, which has mostly focused on studying how the exposome associates with the phenome, needs to take into simultaneous account the other layers of the multiomics universe - from the genome to the metabolome to the microbiome ${ }^{90}$. Another area requiring innovative thinking concerns how to harness big data to better understand human behaviour ${ }^{91}$. Finally, we must consider key questions that are amenable to epidemiologic investigation arising from the major global health challenges: climate change, harmful addictions and mental wellness. What new methodological tools do we need to answer these questions?

Epidemiologists must keep trying on new lenses that correct our own siloed myopia.

\section{Gabriel M. Leung is Helen and Francis} Zimmern Professor in Population Health at WHO Collaborating Centre for Infectious Disease Epidemiology and Control, School of Public Health, LKS Faculty of Medicine, The University of Hong Kong; Chief Scientific Officer at Laboratory of Data Discovery for Health, Hong Kong Science and Technology Park; and Dean of Medicine at the University of Hong Kong, Hong Kong Special Administrative Region, China.

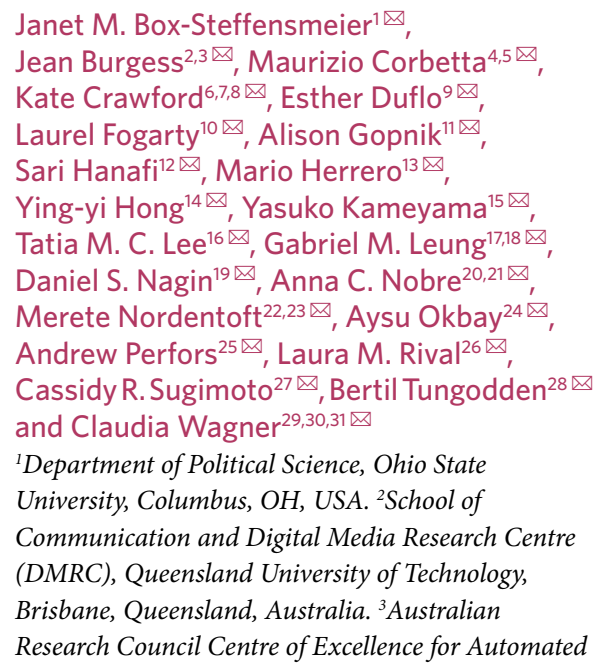

Jean Burgess ${ }^{2,3 凶}$, Maurizio Corbetta ${ }^{4,5 凶}$ Kate Crawford ${ }^{6,7,8} \times$, Esther Duflo ${ }^{9 凶}$, Laurel Fogarty ${ }^{10 凶}$, Alison Gopnik ${ }^{11 凶}$ Sari Hanafi ${ }^{12 凶}$, Mario Herrero ${ }^{13 凶}$ Ying-yi Hong ${ }^{14 凶}$, Yasuko Kameyama ${ }^{15 凶}$, Tatia M. C. Lee ${ }^{16 凶}$, Gabriel M. Leung ${ }^{17,18 凶}$ Daniel S. Nagin ${ }^{19 凶}$, Anna C. Nobre $20,21 \bowtie$, Merete Nordentoft $22,23 凶$, Aysu Okbay ${ }^{24 凶}$ Andrew Perfors ${ }^{25}$, Laura M. Rival ${ }^{26 \bowtie}$ Cassidy R. Sugimoto ${ }^{27 凶}$, Bertil Tungodden ${ }^{28} \bowtie$ and Claudia Wagner 29,30,31

${ }^{1}$ Department of Political Science, Ohio State University, Columbus, OH, USA. ${ }^{2}$ School of Communication and Digital Media Research Centre Brisbane, Queensland, Australia. ${ }^{3}$ Australian Research Council Centre of Excellence for Automated
Decision-Making and Society $(A D M+S)$, Melbourne, Victoria, Australia. ${ }^{4}$ Department of Neuroscience and Padova Neuroscience Center (PNC), University of Padova, Padova, Italy. ${ }^{5}$ Venetian Institute of Molecular Medicine (VIMM), Padova, Italy. ${ }^{6}$ Annenberg School for Communication and Journalism, University of Southern California, Los Angeles, CA, USA. ${ }^{7}$ Microsoft Research New York, New York, NY, USA. ${ }^{8}$ École Normale Supérieure,

Paris, France. ${ }^{9}$ Department of Economics, Massachusetts Institute of Technology, Cambridge, MA, USA. ${ }^{10}$ Department of Human Behavior, Ecology, and Culture, Max Planck Institute for Evolutionary Anthropology, Leipzig, Germany. ${ }^{11}$ Department of Psychology, University of California at Berkeley, Berkeley, CA, USA. ${ }^{12}$ American University of Beirut, Beirut, Lebanon. ${ }^{13}$ Department of Global Development, College of Agriculture and Life Sciences and Cornell Atkinson Center for Sustainability, Cornell University, Ithaca, NY, USA. ${ }^{14}$ Department of Management, The Chinese University of Hong Kong, Hong Kong, Hong Kong Special Administrative Region, China. ${ }^{15}$ Center for Social and Environmental Systems Research, Social Systems Division, National Institute for Environmental Studies, Tsukuba, Japan. ${ }^{16}$ State Key Laboratory of Brain and Cognitive Sciences and Laboratory of Neuropsychology and Human Neuroscience, The University of Hong Kong, Hong Kong, Hong Kong Special Administrative Region, China. ${ }^{17}$ WHO Collaborating Centre for Infectious Disease Epidemiology and Control, School of Public Health, LKS Faculty of Medicine, The University of Hong Kong, Hong Kong, Hong Kong Special Administrative Region, China. ${ }^{18}$ Laboratory of Data Discovery for Health, Hong Kong Science and Technology Park, Hong Kong, Hong Kong Special Administrative Region, China. ${ }^{19} \mathrm{Heinz}$ College of Information Systems and Public Policy, Carnegie Mellon University, Pittsburgh, PA, USA. ${ }^{20}$ Department of Experimental Psychology, University of Oxford, Oxford, UK. ${ }^{21}$ Oxford Centre for Human Brain Activity, Wellcome Centre for Integrative Neuroimaging, Department of Psychiatry, University of Oxford, Oxford, UK. ${ }^{22}$ CORE - Copenhagen Research Centre for Mental Health, Mental Health Centre Copenhagen, Copenhagen University Hospital, Copenhagen, Denmark. ${ }^{23}$ Department of Clinical Medicine, Faculty of Health and Medical Sciences, University of Copenhagen, Copenhagen, Denmark. ${ }^{24}$ Department of Economics, School of Business and Economics, Vrije Universiteit Amsterdam, Amsterdam, The Netherlands. ${ }^{25}$ Complex Human Data Hub, University of Melbourne, Melbourne, Victoria, Australia. ${ }^{26}$ ODID and SAME, University of Oxford, Oxford, UK. ${ }^{27}$ School of Public Policy, Georgia Institute of Technology, Atlanta, GA, USA. ${ }^{28}$ Centre of Excellence FAIR, NHH Norwegian School of Economics, Bergen, Norway. ${ }^{29}$ GESIS - Leibniz Institute for the Social Sciences, Köln, Germany. ${ }^{30}$ RWTH Aachen University, Aachen, Germany. ${ }^{31}$ Complexity Science Hub Vienna, Vienna, Austria.

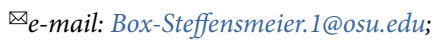


jean.burgess@qut.edu.au; maurizio.corbetta@unipd.it; kate.crawford@usc.edu; eduflo@mit.edu; laurel_fogarty@eva.mpg.de; gopnik@berkeley.edu; Sh41@aub.edu.lb; mario.herrero@cornell.edu; yyhong@cuhk.edu.hk; ykame@nies.go.jp; tmclee@hku.hk; gmleung@hku.hk; dn03@andrew.cmu.edu; kia.nobre@psy.ox.ac.uk; merete.nordentoft@regionh.dk; a.okbay@vu.nl; perfors@gmail.com; laura.rival@anthro.ox.ac.uk; sugimoto@gatech.edu; Bertil.Tungodden@nhh.no; claudia.wagner@gesis.org

Published online: 27 January 2022 https://doi.org/10.1038/s41562-021-01275-6

\section{References}

1. Bahdanau, D., Cho, K. \& Bengio, Y. Neural machine translation by jointly learning to align and translate. Preprint at https://arxiv. org/abs/1409.0473 (2014)

2. Jumper, J. et al. Nature 596, 583-589 (2021)

3. Dwork, C., Hardt, M., Pitassi, T., Reingold, O. \& Zemel, R. Fairness through awareness. In Proc. 3rd Innovations in Theoretical Computer Science Conference, 214-226 (Association for Computing Machinery, 2012).

4. Chouldechova, A. \& Roth, A. Commun. ACM 63, 82-89 (2020).

5. Hara, K. et al. A data-driven analysis of workers' earnings on Amazon Mechanical Turk. In Proc. 2018 CHI Conf. on Human Factors in Computing Systems, 1-14 (Association for Computing Machinery, 2018).

6. Strubell, E., Ganesh, A. \& McCallum, A. Energy and policy considerations for deep learning in NLP. Preprint at https://arxiy. org/abs/1906.02243 (2019).

7. Rival, L. Anthropol. Today 37, 9-12 (2021).

8. Carey, J. W. Communication as Culture: Essays on Media and Society (Unwin Hyman, 1985).

9. Williams, R. Television: Technology and Cultural Form (Fontana, 1974).

10. Helmond, A. Soc. Media Soc. 1, https://doi.org/10.1177/ 2056305115603080 (2015).

11. Rambukkana, N. (Ed.). Hashtag Publics: The Power and Politics of Discursive Networks (Peter Lang, 2015).

12. Burrell, J. Big Data Soc. 3, 1-12 (2016).

13. Bruns, A. et al. Facebook shuts the gate after the horse has bolted, and hurts real research in the process. Internet Policy Review, https://go.nature.com/3IP2xYr (25 April 2018).

14. Article 19. EU: Stop platforms from suppressing public interest research. article.19.org, https://go.nature.com/3DTWgXT (13 September 2021).

15. Burgess, J. et al. Critical simulation as hybrid digital method for exploring the data operations and vernacular cultures of visual social media platforms. Preprint at https://doi.org/10.31235/ osf.io/2cwsu (2021).

16. Rieder, B. \& Hofmann, J. Internet Policy Review 9, 1-28 (2020). 17. Vosoughi, S., Roy, D. \& Aral, S. Science 359, 1146-1151 (2018). 18. Hussein, E., Juneja, P. \& Mitra, T. Measuring misinformation in video search platforms: an audit study on YouTube. In Proc. of the ACM on Human-Computer Interaction 4 (CSCW1) (eds. Lampe C. et al.) 1-27 (Association for Computing Machinery, 2020).

19. Lazer, D. M. J. et al. Science 369, 1060-1062 (2020).

20. Wagner, C. et al. Nature 595, 197-204 (2021).

21. Kosse, F., Deckers, T., Pinger, P., Schildberg-Hörisch, H. \& Falk, A. J. Polit. Econ. 128, 434-467 (2020).
22. Cappelen, A., List, J., Samek, A. \& Tungodden, B. J. Polit. Econ 128, 2739-2758 (2020)

23. Alan, S., Boneva, T. \& Ertac, S. Q. J. Econ. 134, 1121-1162 (2019).

24. Almås, I., Cappelen, A. \& Tungodden, B. J. Polit. Econ. 128, 1753-1788 (2020)

25. Müller, D. \& Renes, S. Soc. Choice Welfare 56, 679-711 (2021).

26. Falk, A. et al. Q. J. Econ. 133, 1645-1692 (2018).

27. Almås, I., Cappelen, A., Sørensen, E. Ø. \& Tungodden, B. Global evidence on the selfish rich inequality hypothesis. Proc. Natl Acad. Sci. USA (in the press).

28. Henrich, J., Root, H. \& Henrich, J. The WEIRDest People in the World: How the West Became Psychologically Peculiar and Particularly Propserous (Farrar, Strauss, and Giroux, 2020).

29. Gelfand, M. J. et al. Lancet Planet. Health 5, e135-e144 (2021).

30. Lu, J. G., Jin, P. \& English, A. S. Proc. Natl Acad. Sci. USA 118, e2021793118 (2021).

31. Hong, Y. Y., Morris, M. W., Chiu, C. Y. \& Benet-Martínez, V. Am. Psychol. 55, 709-720 (2000)

32. Chan, H.-W. et al. Polit. Psychol. 42, 767-793 (2021).

33. Gollwitzer, A. et al. Nat. Hum. Behav. 4, 1186-1197 (2020).

34. de Solla Price, D. Little Science, Big Science (Columbia Univ. Press, 1963).

35. Mauss, M. 1925. in Sociologie et Anthropologie (Réédition 1978) 143-275 (Presses Universitaires de France).

36. Darwin, C. The Descent of Man, and Selection in Relation to Sex (Penguin 2004) (reprint of 2nd edn, 1879).

37. Mesoudi, A. Proc. Natl Acad. Sci. USA 114, 7853-7860 (2017).

38. Cavalli-Sforza, L. \& Feldman, M. W. Theor. Popul. Biol. 4 42-55 (1973).

39. Cavalli-Sforza, L. L. \& Feldman, M. W. Am. J. Hum. Genet. 25, 618-637 (1973).

40. Cavalli-Sforza, L. L. \& Feldman, M. W. Cultural Transmission and Evolution (Princeton Univ. Press, 1981).

41. Richerson, P. J. \& Boyd, R. J. Social Biological Syst. 1, 127-154 (1978).

42. Boyd, R. \& Richerson, P. J. Culture and the Evolutionary Process (Chicago Univ. Press, 1985).

43. Shennan, S. J. Camb. Archaeol. J. 11, 5-16 (2001).

44. Strimling, P., Sjöstrand, J., Enquist, M. \& Eriksson, K. Theor. Popul. Biol. 76, 77-83 (2009).

45. Aoki, K., Lehmann, L. \& Feldman, M. W. Theor. Popul. Biol. 79, 192-202 (2011).

46. Kandler, A. \& Laland, K. N. Theor. Popul. Biol. 76, 59-67 (2009).

47. Laland, K. N. \& O’Brien, M. J. Biol. Theory 6, 191-202 (2011)

48. Richerson, P. J. \& Boyd, R. Not By Genes Alone: How Culture Transformed Human Evolution (Univ. Chicago Press, 2005).

49. Kandler, A. \& Powell, A. Phil. Trans. R. Soc. Lond. B 373, 20170056 (2018).

50. Kandler, A., Wilder, B. \& Fortunato, L. R. Soc. Open Sci. 4, 170949 (2017)

51. McElreath, R. et al. Phil. Trans. R. Soc. Lond. B 363, 3515-3528 (2008)

52. Turkheimer, E. Curr. Dir. Psychol. Sci. 9, 160-164 (2000).

53. Polderman, T. J. C. C. et al. Genet. 47, 702-709 (2015).

54. Visscher, P. M. et al. Am. J. Hum. Genet. 101, 5-22 (2017)

55. Karlsson Linnér, R. et al. Nat. Genet. 51, 245-257 (2019).

56. Lee, J. J. et al. Nat. Genet. 50, 1112-1121 (2018).

57. Liu, M. et al. Nat. Genet. 51, 237-244 (2019).

58. Wray, N. R., Goddard, M. E. \& Visscher, P. M. Genome Res. 17 1520-1528 (2007).

59. The International Schizophrenia Consortium. Nature 460, 748-752 (2009)

60. Becker, J. et al. Nat. Hum. Behav. (2021).

61. Belsky, D. W. et al. Psychol. Sci. 27, 957-972 (2016).

62. Young, A. I., Benonisdottir, S., Przeworski, M. \& Kong, A. Science 365, 1396-1400 (2019)
63. Barcellos, S. H., Carvalho, L. S. \& Turley, P. Proc. Natl Acad. Sci. USA 115, E9765-E9772 (2018).

64. Papageorge, N. W. \& Thom, K. J. Eur. Econ. Assoc. 18, 1351-1399 (2020).

65. DiPrete, T. A., Burik, C. A. P. \& Koellinger, P. D. Proc. Natl Acad. Sci. USA 115, E4970-E4979 (2018).

66. Rietveld, C. A. et al. Science 340, 1467-1471 (2013).

67. Kong, A., Benonisdottir, S. \& Young, A. I. Family analysis with Mendelian imputations. Preprint at https://doi.org/10.1101/ 2020.07.02.185181 (2020).

68. Howe, L. J. et al. Within-sibship GWAS improve estimates of direct genetic effects. Preprint at https://doi.org/10.1101/ 2021.03.05.433935 (2021).

69. Martin, A. R. et al. Nat. Genet. 51, 584-591 (2019).

70. Nuffield Council on Bioethics. Genetics and Human Behaviour The Ethical Context (Nuffield Council on Bioethics, 2002).

71. Redcay, E. \& Schilbach, L. Nat. Rev. Neurosci. 20, 495-505 (2019).

72. Roeckner, A. R., Oliver, K. I., Lebois, L. A. M., van Rooij, S. J. H. \& Stevens, J. S. Transl. Psychiatry 11, 508 (2021).

73. Sevgi, M., Diaconescu, A. O., Henco, L., Tittgemeyer, M. \& Schilbach, L. Biol. Psychiatry 87, 185-193 (2020).

74. Wittmann, M. K. et al. Neuron 91, 482-493 (2016).

75. Gao, M. et al. Neuroimage 223, 117290 (2020).

76. Lee, K. M., Ferreira-Santos, F. \& Satpute, A. B. Neurosci. Biobehav. Rev. 131, 211-228 (2021).

77. Olesen, J., Gustavsson, A., Svensson, M., Wittchen, H. U. \& Jönsson, B. Eur. J. Neurol. 19, 155-162 (2012).

78. Corbetta, M., Siegel, J. S. \& Shulman, G. L. Cortex 107, 229-237 (2018)

79. Griffis, J. C., Metcalf, N. V., Corbetta, M. \& Shulman, G. L. Cell Rep. 28, 2527-2540 (2019).

80. Deco, G. et al. J. Neurosci. 33, 11239-11252 (2013).

81. Patel, V. et al. Lancet 392, 1553-1598 (2018).

82. Correll, C. U. et al. JAMA Psychiatry 75, 555-565 (2018).

83. Petersen, L. et al. Br. Med. J. 331, 602 (2005)

84. Hastrup, L. H. et al. Br. J. Psychiatry 202, 35-41 (2013).

85. Secher, R. G. et al. Schizophr. Bull. 41, 617-626 (2015).

86. Albert, N. et al. Br. Med. J. 356, i6681 (2017).

87. Levitt, T. Harv. Bus. Rev. 38, 45-56 (1960).

88. Davey Smith, G. \& Ebrahim, S. Int. J. Epidemiol. 32, 1-22 (2003).

89. Callaway, E. 'Randomistas' who used controlled trials to fight

poverty win economics Nobel. Nature, https://www.nature.com/ articles/d41586-019-03125-y (14 October 2019).

90. Topol, E. J. Cell 157, 241-253 (2014).

91. Subrahmanian, V. S. \& Kumar, S. Science 355, 489 (2017).

Competing interests

The authors declare no competing interests.

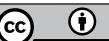

Open Access This article is licensed under a Creative Commons Attribution 4.0 International License, which permits use, sharing, adaptation, distribution and reproduction in any medium or format, as long as you give appropriate credit to the original author(s) and the source, provide a link to the Creative Commons license, and indicate if changes were made. The images or other third party material in this article are included in the article's Creative Commons license, unless indicated otherwise in a credit line to the material. If material is not included in the article's Creative Commons license and your intended use is not permitted by statutory regulation or exceeds the permitted use, you will need to obtain permission directly from the copyright holder. To view a copy of this license, visit http:// creativecommons.org/licenses/by/4.0/. 